\title{
Cost Analysis of the Addition of Hyperacute Magnetic Resonance Imaging for Selection of Patients for Endovascular Stroke Therapy
}

\author{
Seby John ${ }^{a}$ Nicolas R. Thompson ${ }^{b, e}$ Terry Lesko ${ }^{c}$ Nancy Papesh ${ }^{c}$ \\ Nancy Obuchowski ${ }^{b}$ Dan Tomic ${ }^{c}$ Dolora Wisco ${ }^{a}$ Zeshaun Khawaja ${ }^{a}$ \\ Ken Uchino ${ }^{a}$ Shumei Man ${ }^{a}$ Esteban Cheng-Ching ${ }^{a}$ \\ Gabor Toth $^{a}$ Thomas Masaryk $^{d}$ Paul Ruggieri ${ }^{d}$ Michael Modic $^{c}$ \\ Muhammad Shazam Hussain ${ }^{a}$ \\ ${ }^{a}$ Cerebrovascular Center, Neurological Institute, ${ }^{b}$ Department of Quantitative Health \\ Sciences, ${ }^{\mathrm{c}}$ Neurological Institute, ${ }^{\mathrm{d}}$ Imaging Institute, and ${ }^{\mathrm{e}}$ Neurological Institute Center for \\ Outcomes Research and Evaluation, Cleveland Clinic, Cleveland, OH, USA
}

\section{Keywords}

Ischemic stroke $\cdot$ Magnetic resonance imaging $\cdot$ Patient selection $\cdot$ Cost analysis

\begin{abstract}
Background and Purpose: Patient selection is important to determine the best candidates for endovascular stroke therapy. In application of a hyperacute magnetic resonance imaging (MRI) protocol for patient selection, we have shown decreased utilization with improved outcomes. A cost analysis comparing the pre- and post-MRI protocol time periods was performed to determine if the previous findings translated into cost opportunities. Materials and Methods: We retrospectively identified individuals considered for endovascular stroke therapy from January 2008 to August 2012 who were $\leq 8 \mathrm{~h}$ from stroke symptoms onset. Patients prior to April 30, 2010 were selected based on results of the computed tomography/ computed tomography angiography alone (pre-hyperacute), whereas patients after April 30, 2010 were selected based on results of MRI (post-hyperacute MRI). Demographic, outcome, and financial information was collected. Log-transformed average daily direct costs were regressed on time period. The regression model included demographic and clinical covariates as potential confounders. Multiple imputation was used to account for missing data. Results: We identified 267 patients in our database (88 patients in pre-hyperacute MRI period, 179 in hyperacute MRI protocol period). Patient length of stay was not significantly different in the hyperacute MRI protocol period as compared to the pre-hyperacute MRI period (10.6 vs. 9.9 days, $p<0.42$ ). The median of average daily direct costs was reduced by $24.5 \%$ ( $95 \%$ confi-
\end{abstract}

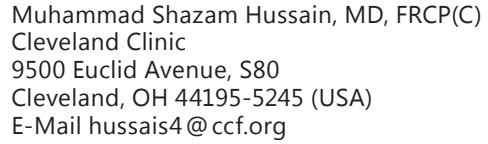


dence interval 14.1-33.7\%, $p<0.001)$. Conclusions: Use of the hyperacute MRI protocol translated into reduced costs, in addition to reduced utilization and better outcomes. MRI selection of patients is an effective strategy, both for patients and hospital systems.

(C) 2017 S. Karger AG, Basel

\section{Introduction}

Rising healthcare costs have become a national concern as the payor climate shifts from passive payment (fee-for-service) to purchasers of higher quality and affordable care (valuebased care) [1]. This has pressured institutions to seek strategies that improve outcomes while reducing costs. The American Heart Association ${ }^{\circledR}$ projects stroke-related costs to triple from USD 71.6 billion to USD 184.1 billion by 2030 [2]. Of those costs, the vast majority is attributed to patients who are left with severe dependency from stroke. Endovascular stroke therapy (EST) has gained much traction over the years as an intervention to improve outcomes in patients with intracranial large vessel occlusion (LVO) as only $25 \%$ of patients with LVO experience favorable outcomes at 90 days [3]. Recent clinical trials have confirmed the efficacy of intra-arterial stroke therapy for acute ischemic stroke [4-7]. The high costs of endovascular therapy are thought to be offset by the long-term savings of reduced skilled needs and institutional or home rehabilitative care. No consensus presently exists as to the best strategy for patient selection for acute endovascular ischemic stroke therapy. Increasingly, more centers are moving toward advanced imaging-based selection. Magnetic resonance imaging (MRI) diffusion-weighted imaging (DWI) offers the advantage over computed tomography (CT) of clearly identifying cerebral infarct [8, 9], though concerns of time delays exist [10]. In addition, many hospitals are concerned about the additional cost of MRI in the stroke process. At our center, we have implemented a hyperacute MRI protocol to aid in patient selection and we have reported this strategy to reduce utilization of EST by nearly half, with improvement in outcome [11]. We hypothesized that despite the increased cost of MRI in the selection process, the decreased utilization of EST and improved outcomes should translate into decreased overall cost. A cost analysis of comparing the pre-hyperacute MRI protocol period and the hyperacute MRI protocol period was performed.

\section{Methods}

\section{Subjects}

From January 2008 to August 2012, we retrospectively identified patients older than 18 years who presented to our emergency department or transferred from other hospitals with acute ischemic stroke $\leq 8$ $\mathrm{h}$ since last seen well who were considered for EST from our prospectively maintained database. All patients received standard of care, including IV tPA if eligible. The Institutional Review Board approved this study.

From a prospectively collected database maintained in REDCap [12], the following baseline clinical characteristics and treatment modalities were collected: demographic data, stroke risk factors, initial National Institutes of Health Stroke Scale (NIHSS) and baseline pre-stroke modified Rankin Scale (mRS) scores, time of last known well, pretreatment imaging, endovascular treatment modality (intra-arterial thrombolysis infusion, glycoprotein IIb/IIIa inhibitors, mechanical thrombectomy devices used), and 30-day mRS outcome data (performed by either in-person or telephone follow-up).

Pre-Hyperacute MRI Protocol

Patients in the pre-hyperacute MRI period presented from January 1, 2008 to April 29, 2010. They were selected for EST on the basis of clinical examination with initial NIHSS $\geq 8$, non-contrast CT of the head that did not show an acute infarct in $>1 / 3$ of the vessel territory, and CT angiography (CTA) showing a corresponding LVO. Further information on imaging parameters is available in the Data Supplement of the original study. 
Hyperacute MRI Protocol

Patients in the hyperacute MRI period presented from April 30, 2010 to August 31, 2012. They were selected for EST on the basis of previously stated CT/CTA criteria and the result of the pretreatment brain MRI. Patients with a clinically large stroke were taken directly for hyperacute MRI after CTA revealed the presence of an LVO. Those with a contraindication to CTA, such as an increased creatinine or allergy to iodine contrast, or those with nondiagnostic CTA whose clinical examination was consistent with an LVO were taken for hyperacute MRI, and in these instances, an MRA of the head and neck was obtained. To minimize time to EST, as soon as a clinical examination with baseline (NIHSS $\geq 8$ ) and concomitant LVO was found on CTA, the endovascular team was activated. On the basis of MRI results, those patients thought to have either clinicalDWI mismatch (clinical deficits out of proportion to DWI lesion, approximately $<70$-mL volume core infarct by visual inspection) or DWI-perfusion weighted imaging mismatch (by visual inspection of perfusion maps) were immediately taken for EST.

\section{Statistical Analysis}

Patient Population

Summary statistics (e.g., means, standard deviations, frequencies, percentages, etc.) of demographic and clinical variables were calculated for patients overall and within the two groups (pre-hyperacute MRI protocol and hyperacute MRI protocol). Comparisons were made between the groups using $t$ tests for continuous variables and $\chi^{2}$ tests for categorical variables. Non-parametric tests (e.g., permutation test, Fisher exact test) were used when the assumptions of parametric tests were questionable. For all analyses, we used $\mathrm{R}$ (version 2.15.3) [13], and $p$ values $<0.05$ were considered significant.

\section{Cost Analysis}

Financial direct costs were obtained via our enterprise's cost accounting systems. Cost data for patients prior to January 1, 2010 is maintained within the legacy system TSI (Elcipsys Corp). In 2010, the enterprise launched the EPSi cost accounting platform, where data, both financial and operational in nature, for encounters occurring after December 31, 2009 are maintained and updated. Costs were adjusted for inflation using the U.S. Bureau of Labor statistics Consumer Price Index for All-Urban Consumers (CPI-U).

In order to determine if direct costs were significantly lower in the post-MRI protocol period versus the MRI protocol period, we created a multiple linear regression model. The dependent variable was the logarithm of average daily direct cost. Average daily direct cost was computed by dividing the sum of fixed and variable direct costs by the patient's length of hospital stay (time from admission to discharge). A log-transformation was necessary to mitigate the issues of non-normal residuals and non-constant variance. The independent variable of interest was an indicator for time period defined as 0 if the patient was admitted before April 30, 2010 and 1 if the patient was admitted on or after April 30, 2010. We adjusted for the following patient-level covariates: age at admission (in years), gender (male, female), race (white, black, other), insurance status (Medicare, Medicaid, private/other), stroke etiology (cardioembolism, large artery atherosclerosis, other), IV tPA (yes, no), pre-stroke mRS, and NIHSS score at admission. Because we used the logarithmic transformation on average daily cost, exponentiation of the coefficient for the time period indicator provides an estimate of the ratio of the median of the average daily direct costs for the two groups. From this we computed an estimate of the reduction in the median of average daily direct cost along with a $95 \%$ confidence interval (CI).

\section{Missing Data}

We anticipated varying amounts of missing data for the variables of interest. For all variables, we calculated the percentage of missing values for both groups.

For the cost analysis, we used multiple imputation [14] to create and analyze 10 imputed datasets. Incomplete variables were imputed under fully conditional specification [15]. Calculations were done in $\mathrm{R}$ 2.15.3 using the default settings of the MICE 2.13 package [16]. Model parameters were estimated with multiple linear regression applied to each imputed dataset separately. These estimates and their standard errors were combined using Rubin's rules. We also performed complete case and sensitivity analyses to compare results to those obtained under multiple imputation.

We also examined change in cost from the pre-hyperacute MRI period to hyperacute MRI period by institute (e.g., Imaging, Nursing, Neurological, Pharmacy, Anesthesiology). We performed Mann-Whitney U tests to determine if costs were significantly different in the two time periods for each institute. To adjust for multiple comparisons, we used Holm's method [17]. 
John et al.: Cost Analysis of the Addition of Hyperacute Magnetic Resonance Imaging for Selection of Patients for Endovascular Stroke Therapy

Table 1. Summary statistics for all patients and stratified by pre/post MRI protocol group

\begin{tabular}{|c|c|c|c|c|}
\hline & All patients & Before MRI protocol & After MRI protocol & $p$ value \\
\hline$n$ & 267 & 88 & 179 & \\
\hline Age, years & $70.4 \pm 14.9$ & $70.2 \pm 14.8$ & $70.5 \pm 14.9$ & 0.8636 \\
\hline Gender & & & & 0.9871 \\
\hline Male & $117(43.8)$ & $38(43.2)$ & $79(44.1)$ & \\
\hline Female & $150(56.2)$ & $50(56.8)$ & $100(55.9)$ & \\
\hline Ethnicity & & & & 0.5587 \\
\hline Caucasian & $191(72.6)$ & $63(71.6)$ & $128(73.1)$ & \\
\hline African-American & $61(23.2)$ & $22(25)$ & $39(22.3)$ & \\
\hline Asian & $1(0.4)$ & $0(0.0)$ & $1(0.6)$ & \\
\hline Hispanic & $3(1.1)$ & $2(2.3)$ & $1(0.6)$ & \\
\hline Other & $7(2.7)$ & $1(1.1)$ & $6(3.4)$ & \\
\hline Insurance & & & & 0.2265 \\
\hline Medicare & $178(66.7)$ & $56(63.6)$ & $122(68.2)$ & \\
\hline Medicaid & $19(7.1)$ & $4(4.5)$ & $15(8.4)$ & \\
\hline Private/other & $70(26.2)$ & $28(31.8)$ & $42(23.5)$ & \\
\hline Stroke etiology & & & & 0.1639 \\
\hline Cardioembolism & $137(51.5)$ & $53(60.2)$ & $84(47.2)$ & \\
\hline Large artery & $58(21.8)$ & $18(20.5)$ & $40(22.5)$ & \\
\hline Cryptogenic & 47 (17.7) & $14(15.9)$ & $33(18.5)$ & \\
\hline Dissection & $3(1.1)$ & $1(1.1)$ & $2(1.1)$ & \\
\hline IV tPA, yes & $116(43.4)$ & $32(36.4)$ & $84(46.9)$ & 0.1447 \\
\hline Pre-stroke Rankin score & & & & 0.3358 \\
\hline 0 & $122(58.9)$ & $14(50.0)$ & $108(60.3)$ & \\
\hline 1 & $44(21.3)$ & $5(17.9)$ & $39(21.8)$ & \\
\hline 2 & $16(7.7)$ & $5(17.9)$ & $11(6.1)$ & \\
\hline 3 & $16(7.7)$ & $3(10.7)$ & $13(7.3)$ & \\
\hline 4 & $7(3.4)$ & $1(3.6)$ & $6(3.4)$ & \\
\hline 5 & $2(1.0)$ & $0(0.0)$ & $2(1.1)$ & \\
\hline Admission NIHSS score & $16 \pm 6.7$ & $16.4 \pm 6.5$ & $15.8 \pm 6.8$ & 0.5019 \\
\hline Admission CT ASPECTS score & $8(7-10)$ & $8(7-9)$ & $9(6-10)$ & 0.97 \\
\hline Length of stay, days & $10.1 \pm 7.6$ & $10.6 \pm 7.2$ & $9.9 \pm 7.8$ & 0.4206 \\
\hline
\end{tabular}

Values are mean \pm standard deviation, $n(\%)$, or median (interquartile range).

\section{Results}

\section{All Patients}

Two hundred and sixty-seven patients (88 [33.0\%] in the pre-hyperacute period and 179 $[67.0 \%]$ in the hyperacute period) were included in our study analysis. Summary statistics for all patients and stratified by time period are provided in Table 1. Overall, the average age was $70.4 \pm 14.9$ years, $56.2 \%$ were female, and $72.6 \%$ were Caucasian. Among the variables listed in Table 1, there were no significant differences between the two groups. However, a close examination of the counts of pre-stroke $\mathrm{mRS}$ in the pre-hyperacute MRI protocol group reveals that only 28 of the 88 patients had a pre-stroke mRS, while all 179 patients in the post-MRI protocol group had a pre-stroke mRS.

Among the other variables listed in Table 1, we were missing 4 values for race, 1 value for stroke etiology, and 1 value for IV tPA. We had complete data for all other variables in Table 1. Thus, for the multiple linear regression model, the only variable with a substantial amount of missing data was pre-stroke mRS. There was no significant difference in stroke severity measured by mean NIHSS score, baseline CT ASPECTS, or baseline mRS between the 2 groups. In the pre-hyperacute period, all but $3(3.4 \%)$ of the 88 patients received EST, 
John et al.: Cost Analysis of the Addition of Hyperacute Magnetic Resonance Imaging for Selection of Patients for Endovascular Stroke Therapy

Fig. 1. Change in median cost by institute and protocol period.

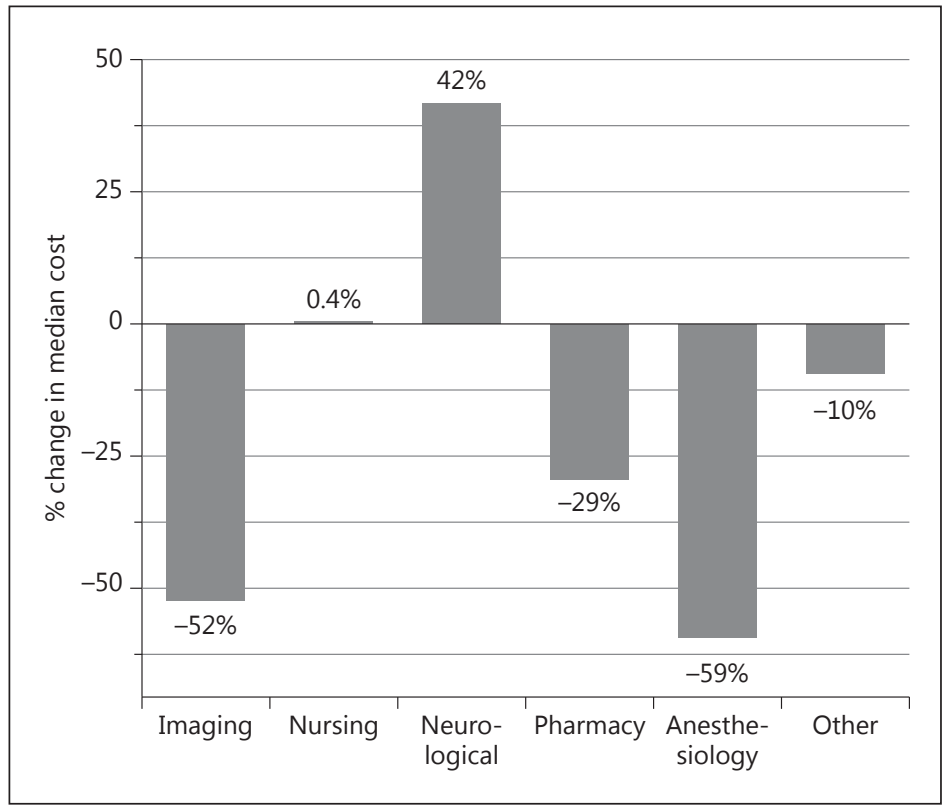

whereas in the hyperacute protocol period, about half of the patients who were thought to be candidates based on CT/CTA and MRI underwent EST, yielding a significant difference from pre- to post-MRI protocol (85 [96.6\%] vs. 92 [51.4\%], respectively, $p \leq 0.001$ ). There was no significant difference in the rates of successful recanalization in the pre- and post-MRI group (42 [49.4\%] vs. 51 [55.4\%], $p=0.40$ ). There was also no significant difference of time from symptom onset to intravenous thrombolysis or successful recanalization between groups. There was also no difference between the time from CT to groin puncture between groups. Rate of post-procedural hemorrhage (parenchymal hematoma -2) was also similar between the pre- and post-hyperacute MRI periods ( 9.8 vs. $5.8 \%, p=0.50$ ).

In terms of outcome data, 30-day mRS was available in 66 of 88 (75\%) of the patients in the pre-hyperacute MRI period compared to 140 of 179 (78\%). Despite decreasing the rate of EST being performed for acute stroke patients with LVO in the hyperacute MRI protocol period, more patients had overall favorable outcome based on 30-day mRS $\leq 2$ (33 [23.6\%] vs. 6 [9.1\%], $p<0.001$ ) and the 30-day mortality was significantly decreased in the hyperacute MRI period (32 [36.3\%] vs. 41 [22.9\%], $p=0.03$ ).

\section{Cost Analysis}

Patient length of stay did not significantly differ in the hyperacute MRI protocol period as compared to the pre-hyperacute MRI period (10.6 vs. 9.9 days, $p<0.42$ ). After adjusting for the effects of age, gender, race, insurance status, stroke etiology, IV tPA administration, pre-stroke mRS, and admission NIHSS score, the median of the average daily direct cost was reduced by $24.5 \%$ (95\% CI $14.1-33.7 \%, p<0.0001)$ from the pre-hyperacute MRI protocol period to the post-hyperacute MRI protocol period. In addition, there was not a significant difference in median costs among patients who achieved successful recanalization in the prehyperacute versus post-hyperacute MRI periods, whether adjusted for confounding variables or not.

Figure 1 displays the change in median cost for each institute by protocol period, and Figure 2 displays the proportion of costs based on institute. Direct costs were significantly lower in the post-protocol period for the imaging and anesthesiology institutes (Holm- 
John et al.: Cost Analysis of the Addition of Hyperacute Magnetic Resonance Imaging for Selection of Patients for Endovascular Stroke Therapy

Fig. 2. Percentage of total cost by institute.

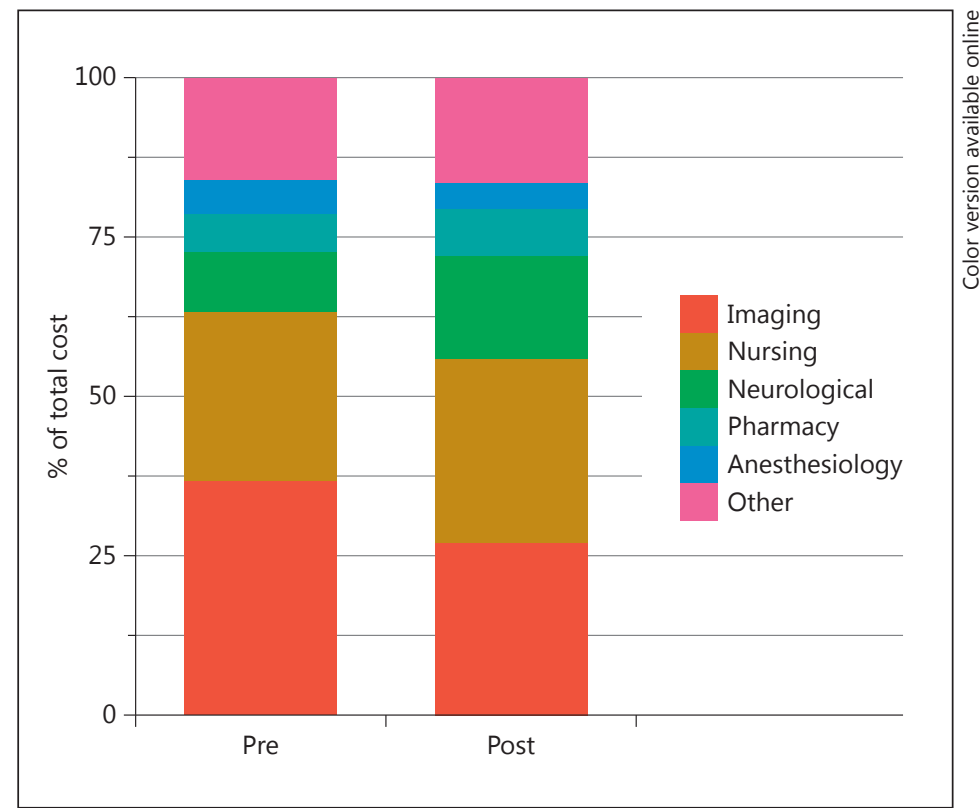

adjusted $p$ value $<0.0001$ for both). No significant change in direct costs for the nursing institutes was found (Holm-adjusted $p$ value $=0.2369$ ). Direct costs were significantly higher in the neurological institutes (Holm-adjusted $p$ value $=0.0054$ ), and significantly lower in pharmacy (Holm-adjusted $p$ value $=0.0397$ ), and all other institutes combined (Holmadjusted $p$ value $=0.0397$ ). In the pre-protocol period, the imaging institute made up about $37 \%$ of the total direct costs, while in the post-protocol period, it made up about $27.4 \%$ of the total direct costs. Direct costs in the neurological institute increased from about 9.5 to $16.5 \%$ of total direct costs from the pre- to the post-hyperacute MRI period.

\section{Sensitivity Analysis}

As noted earlier, 68.2\% (60/88) of pre-hyperacute MRI protocol patients were missing their pre-stroke mRS, while none of the post-hyperacute MRI protocol patients were missing this variable. The above cost analysis, which adjusted for pre-stroke mRS, included imputed values for pre-stroke mRS using multiple imputation. To test the sensitivity of imputed values, we examined two extreme cases: one where all missing pre-stroke mRS would have actually been 0 and one where all pre-stroke mRS would have been 5 . We also examined complete case analysis with all variables used in the model under multiple imputation and a complete case analysis with pre-stroke mRS removed from the model.

When missing pre-stroke mRS were constrained to all be 0 , the reduction in the median of the average daily direct cost dropped to $20.1 \%$ and was still statistically significant ( $95 \%$ CI $9.0-29.9 \%, p=0.0008$ ). When missing pre-stroke mRS were constrained to all be 5 , the reduction in the median of the average daily direct cost dropped to $11.9 \%$ and was no longer statistically significant ( $95 \%$ CI -0.07 to $27.4 \%, p=0.1948$ ).

Although both of these extreme cases reduced the difference in the estimates of direct costs for the two groups, it is unlikely that all of these patients' scores would lie at one of the extremes. The imputation model we created accounted for important information such as age and comorbidity history in creating several sets of variable but plausible values for pre-stroke mRS. 
Under complete case analysis with pre-stroke mRS included in the model, the reduction in the median of the average daily direct cost was $5.8 \%(95 \% \mathrm{CI}+13.8$ to $-22.1 \%, p=0.5305)$. It should be noted that $60 / 88$ patients in the pre-hyperacute group were removed from this analysis. Complete case analysis with pre-stroke mRS excluded from the model yielded results much more similar to the results under multiple imputation. The estimated reduction in the median of the average daily direct cost was $23.2 \%$ (95\% CI $12.8-32.3 \%, p<0.0001)$.

\section{Discussion}

In this cost analysis, the median of the average daily direct costs was reduced by $24.5 \%$ from the pre-hyperacute MRI to the hyperacute MRI period. This finding, along with decreased procedure utilization and improved patient outcomes, suggests that the addition of MRI for patient selection not only benefits patients, but also creates a cost opportunity for hospital systems as well. Particularly in the current environment of increasing health care costs and reducing reimbursements [18], finding better patient selection strategies is critical to the proper application and utilization of therapies.

EST is a resource-intensive treatment. Equipment and personnel costs exist, including the cost of devices for mechanical thrombectomy as well as angiographic radiologic technologists and nursing support. Reducing the overall number of interventions by half will reduce the utilization of these resources. In addition, avoiding interventions in patients with substantial core infarction (who have little to no benefit from the procedure) also reduces post-procedure costs of care. Most patients who receive EST are managed in a neurological intensive care unit (NICU); those who do not receive EST may not require NICU care and this also reduces overall cost. It is also possible that exposing patients with large core infarction to vessel manipulation via intervention and anesthesia (either general anesthesia or sedation) may cause them to look worse after the procedure, prompting more invasive procedures and supportive care. In post-hyperacute MRI protocol implementation, patients were also noted to have improved outcomes. Baseline functional status, stroke severity, procedural success, or complications did not explain this difference. It is likely that patients in the post-hyperacute group required less intensive care after admission and thus were able to move to the next phase of their care more quickly.

We acknowledge that rates of successful recanalization and good outcomes in this cohort are low compared to recently published positive randomized controlled trials. However, techniques and devices used for EST during the majority of the time period in our study were drastically different and less effective at achieving recanalization. In addition, baseline disability among other variables did not necessarily preclude selection of patients for EST.

This analysis only looks at the acute care costs of this patient population. By decreasing stroke disability, we also will decrease long-term costs for stroke. Costs associated with an mRS of 4 or 5 were three times higher than for an mRS of 2 or less and $70 \%$ higher than for an mRS of 3 [19]. Furthermore, $75 \%$ of the cost associated with a 90-day episode of stroke care occurs in the post-acute space rather than during the inpatient hospitalization, and payments for patients going to a skilled nursing facility or acute inpatient rehabilitation are 3-5 times higher than for patients who are discharged directly to home, respectively [20-22]. By reducing immediate disability, the patient should be on a trajectory for also improving cost in the post-acute space.

Overall, the health care system is facing great challenges as cost of care continues to rise and the population is aging, resulting in more individuals requiring care. Protocols that aid in selection, especially for procedures with potential for risk and high cost, are needed to treat those with the best chance for success and avoid intervention in those where there is little to 
John et al.: Cost Analysis of the Addition of Hyperacute Magnetic Resonance Imaging for Selection of Patients for Endovascular Stroke Therapy

no chance for improvement. Continued development of these protocols, both within stroke treatment and for treatment of other diseases, will allow people to obtain the best health care outcomes while containing health care costs.

\section{Disclosure Statement}

The authors have no conflicts of interest to report. No external funding was utilized in the performance of this research or writing of the manuscript.

\section{References}

1 Blasel S, Yukzek Z, Kurre W, et al: Recanalization results after intracranial stenting of atherosclerotic stenoses. Cardiovasc Intervent Radiol 2010;33:914-920.

2 Ovbiagele B, Goldstein LB, Higashida RT, et al: Forecasting the future of stroke in the United States: a policy statement from the American Heart Association and American Stroke Association. Stroke 2013;44:23612375.

3 Furlan A, Higashida R, Wechsler L, et al: Intra-arterial prourokinase for acute ischemic stroke. The PROACT II study: a randomized controlled trial. Prolyse in Acute Cerebral Thromboembolism. JAMA 1999;282:20032011.

4 Berkhemer OA, Fransen PS, Beumer D, et al: A randomized trial of intraarterial treatment for acute ischemic stroke. N Engl J Med 2015;372:11-20.

5 Goyal M, Demchuk AM, Menon BK, et al: Randomized assessment of rapid endovascular treatment of ischemic stroke. N Engl J Med 2015;372:1019-1030.

6 Campbell BC, Mitchell PJ, Kleinig TJ, et al: Endovascular therapy for ischemic stroke with perfusion-imaging selection. N Engl J Med 2015;372:1009-1018.

7 Saver JL, Goyal M, Bonafe A, et al: Stent-retriever thrombectomy after intravenous t-PA vs. t-PA alone in stroke. N Engl J Med 2015;372:2285-2295.

8 Gonzalez RG, Schaefer PW, Buonanno FS, et al: Diffusion-weighted MR imaging: diagnostic accuracy in patients imaged within 6 hours of stroke symptom onset. Radiology 1999;210:155-162.

9 Fiebach JB, Schellinger PD, Jansen O, et al: CT and diffusion-weighted MR imaging in randomized order: diffusion-weighted imaging results in higher accuracy and lower interrater variability in the diagnosis of hyperacute ischemic stroke. Stroke 2002;33:2206-2210.

10 Sheth KN, Terry JB, Nogueira RG, et al: Advanced modality imaging evaluation in acute ischemic stroke may lead to delayed endovascular reperfusion therapy without improvement in clinical outcomes. J Neurointerv Surg 2013;5(suppl 1):i62-i65.

11 Wisco D, Uchino K, Saqqur M, et al: Addition of hyperacute MRI AIDS in patient selection, decreasing the use of endovascular stroke therapy. Stroke 2014;45:467-472.

12 Harris PA, Taylor R, Thielke R, Payne J, Gonzalez N, Conde JG: Research electronic data capture (REDCap) - a metadata-driven methodology and workflow process for providing translational research informatics support. J Biomed Inform 2009;42:377-381.

13 R Development Core Team (2013). R: A Language and Environment for Statistical Computing. R Foundation for Statistical Computing, Vienna, Austria. ISBN 3-900051-07-0, http://www.R-project.org/.

14 Rubin D: Multiple Imputation for Nonresponse in Surveys. New York: John Wiley \& Sons, 1987.

15 Van Buuren S, Brand JPL, Groothuis-Oudshoorn CGM, Rubin DB: Fully conditional specification in multivariate imputation. J Stat Comput Simul 2006;76:1049-1064.

16 Van Buuren S, Groothuis-Oudshoorn CGM: MICE: multivariate imputation by chained equations in R. J Stat Softw 2011;45:1-67.

17 Holm S: A simple sequentially rejective multiple test procedure. Scand J Stat 1979;6:65-70.

18 Schroeder SA, Frist W: Phasing out fee-for-service payment. N Engl J Med 2013;368:2029-2032.

19 Spieler JF, Lanoe JL, Amarenco P: Costs of stroke care according to handicap levels and stroke subtypes. Cerebrovasc Dis 2004;17:134-142.

20 Cohen MA, Tumlinson A: Understanding the state variation in Medicare home health care. The impact of Medicaid program characteristics, state policy, and provider attributes. Med Care 1997;35:618-633.

21 Kane RL, Lin WC, Blewett LA: Geographic variation in the use of post-acute care. Health Serv Res 2002;37: 667-682.

22 Medicare Payment Advisory Commission. Report to the Congress: Medicare Payment Policy. Chapter 4: Post acute care providers: an overview of the issues. March 2006. 